\title{
Real-time feedback control of millimeter-wave polarization for LHD
}

\author{
F. Felici, ${ }^{1, a)}$ T. Goodman, ${ }^{1}$ O. Sauter, ${ }^{1}$ T. Shimozuma, ${ }^{2}$ S. Ito, ${ }^{2}$ Y. Mizuno, ${ }^{2}$ S. Kubo, ${ }^{2}$ and \\ T. Mutoh ${ }^{2}$ \\ ${ }^{1}$ Centre de Recherches en Physique des Plasmas, Association EURATOM-Suisse, École Polytechnique \\ Fédérale de Lausanne (EPFL), 1015 Lausanne, Switzerland \\ ${ }^{2}$ National Institite for Fusion Science (NIFS), Oroshi-cho, Toki 509-5292, Japan
}

(Received 21 August 2008; accepted 1 January 2009; published online 29 January 2009)

\begin{abstract}
Electron cyclotron heating $(\mathrm{ECH})$ is widely used in magnetic fusion devices, and the polarization of the injected millimeter-wave beams plays a crucial role in the propagation and absorption of the beam energy by the plasma. This polarization can be adjusted by grating mirror polarizers placed in the transmission lines which carry the microwaves from the power source to the plasma. In long-pulse devices such as the Large Helical Device (LHD) and ITER, it is desirable to track changes in the plasma and adjust the polarization of the ECH in real time such as to keep the absorption as high as possible and avoid shine-through which may lead to overheating of vessel components. For this purpose a real-time feedback control scheme is envisioned in which a measure of the absorption efficiency can be used to adjust the orientation of the polarizing mirrors toward an optimum. Such a setup has been tested in a low-power test stand as preparation for future implementation in the LHD ECH system. It is shown that a simple search algorithm is efficient and can in principle be used to control either the absorption efficiency or the linear polarization angle. (C) 2009 American Institute of Physics. [DOI: 10.1063/1.3073735]
\end{abstract}

\section{INTRODUCTION}

Waves in the electron cyclotron range of frequencies are routinely used in fusion devices for plasma heating $(\mathrm{ECH})$ and current drive. Since these waves are able to propagate in free space, it is possible to launch them remotely. When they reach the location of the plasma boundary, the waves can couple to electromagnetic waves in the plasma in two modes, commonly referred to as $X$ and $O$ modes. These modes have distinct propagation and absorption characteristics ${ }^{1}$ and, depending on the injection path and the frequencies involved, injection of one of these modes is generally preferable.

The split of power between the two modes in the plasma depends on the polarization of the incoming wave and on the properties (magnetic geometry and density) of the plasmavacuum boundary. In practice, the polarization of the EC wave is set to maximize the absorption. In state-of-the-art experiments worldwide, this is done by preprogramming the positions of a set of polarizing mirrors. For the microwaves created by gyrotrons, which are linearly polarized, a combination of $\lambda / 4$ and $\lambda / 8$ polarizing mirrors can be used to obtain essentially any desired polarization state. This approach is satisfactory when the characteristics of the plasmavacuum interface is known. In particular, if the plasma density rises sharply at the plasma "edge," then a precise location [referred to as the last closed flux surface (LCFS)] can be considered as the interface between the plasma and the vacuum. At this location, the wave can be decomposed into an $X$ and $O$ mode component which propagate independently through the plasma. Therefore, the optimal polarization can be derived from knowledge of the magnetic field

${ }^{a)}$ Electronic mail: federico.felici@epfl.ch. configuration at this position. The required polarization can be a combination of linear and elliptical polarizations depending on the injection angle with respect to the magnetic field.

However, studies of the plasma-vacuum interface on Large Helical Device (LHD) plasmas have shown that there is no such clearly defined edge. Rather, the plasma extends beyond the LCFS into an ergodic layer. Thomson scattering measurements ${ }^{2}$ indicate that the plasma density is nonnegligible in this region. Therefore it is difficult to determine precisely what magnetic field geometry should be considered in calculating the optimal polarization setting. Moreover, it is shown in Ref. 3 that in regions of high magnetic shear and low density, such as at the edge of the LHD and other stellarators, ${ }^{4}$ significant coupling between $O$ and $X$ modes exists. Therefore, variations in time of the plasma density at the ECH beam intersection with the plasma, as well as variations in time of the plasma boundary location, have an effect on the absorption efficiency. This is particularly important in LHD since it is a long-pulse device with the capability of sustaining plasmas for several minutes. Changing the polarization of the incident ECH beam in real time during a plasma discharge would allow to compensate for changes in the plasma properties/beam aiming and maintain maximal absorption efficiency throughout the discharge.

For this purpose, a system for real-time feedback control of the polarization of EC waves is under development at LHD as an extension of the existing ECH control system. ${ }^{5}$ The control of polarization is also relevant for the planned ITER ECH transmission line system, ${ }^{6}$ which will also includes rotating polarizer mirrors. The objective at LHD is to measure the absorption of the EC waves and to adjust the two polarizing mirrors in such a way as to maximize this 


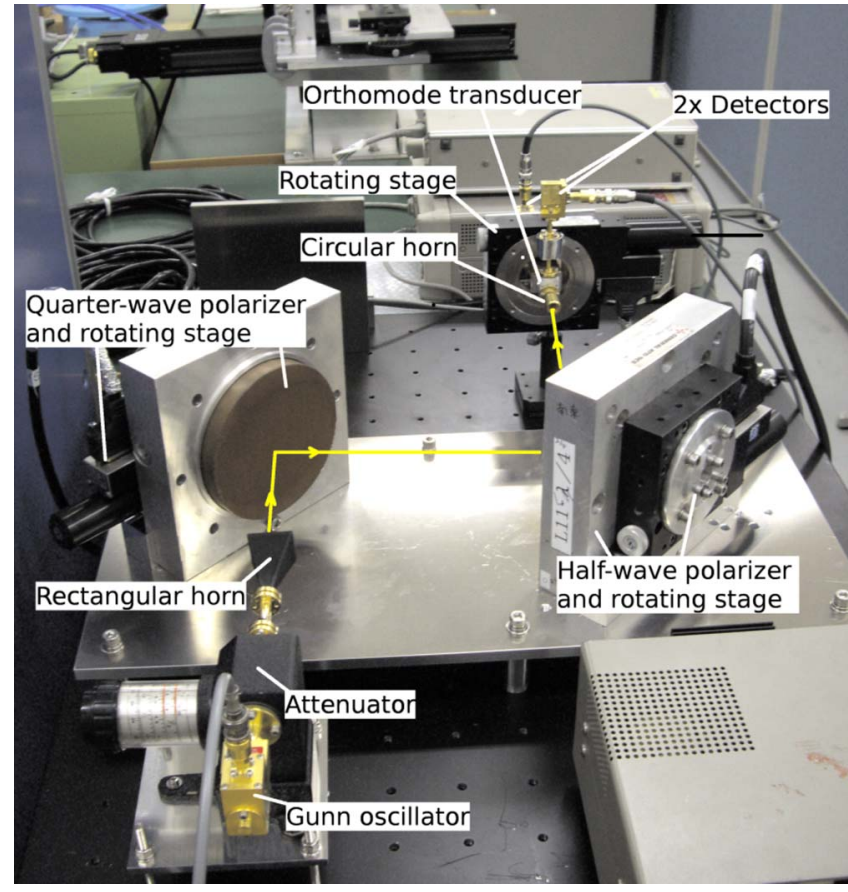

FIG. 1. (Color online) Photograph of the setup in the low-power test stand at NIFS

absorption. As a preliminary step in this development, feedback control experiments have been performed on a lowpower test stand using the same polarizing mirrors as employed in the LHD ECH transmission lines but without the possible complications (arcing, heating, etc.) associated with high power. This paper reports on the results of these experiments and on the control algorithms used for real-time feedback.

This paper is structured as follows. Section II describes the hardware setup employed for the experiments. Section III then illustrates the control strategies and the algorithm employed. Section IV shows the results of the experiments, and finally Sec. V summarizes the results.

\section{HARDWARE SETUP}

This section describes the hardware setup as it has been constructed in the low-power test stand at NIFS in Japan. The setup is shown in Fig. 1. Low-power microwaves are generated by a $84 \mathrm{GHz}$ Gunn oscillator and first pass through an attenuator to a rectangular horn. This horn is set up such that the outgoing wave is linearly polarized with the electric field in the vertical direction. The wave subsequently encounters a $\lambda / 8$ polarizer (quarter-wave plate) and a $\lambda / 4$ polarizer (half-wave plate), both at $45^{\circ}$ incidence angle. The wave reflected off the last polarizing mirror is received by a circular horn antenna. This circular horn is connected to an orthomode transducer which feeds the two orthogonal components to two crystal detectors. The relative calibration of the two crystal detectors was determined by rotating the detector assembly $90^{\circ}$ and ensuring that the measured signals matched.

Both polarizing mirror gratings (Acquired from General Atomics, San Diego, CA) are mounted on Newport 450CC rotating stages. The crystal detectors and horn assembly is also mounted on a third, identical rotating stage. This allows the rotation of the receiver to capture different components of the wave's electric field. When the rotating stages are at $0^{\circ}$, the polarizer mirrors have the gratings in vertical direction and the two detectors measure vertical and horizontal polarization components, respectively. The rotating stages are limited to move at a maximum of $2 \%$.

All the rotating stages are controlled by a Newport ESP300 motion controller which has a built-in feedforwardproportional-integral-derivative controller. It is possible to send motion commands to set a desired polarizer angle directly to this motion controller, which takes care of the positioning. Communication with the motion controller is achieved using a general purpose interface bus (IEEE-488) interface connected to a personal computer (PC) using an ethernet connection. The voltage signal from the crystal detectors is directly acquired using a National Instruments NI6218 USB device connected to the same PC running Windows XP and LABWINDOWS/CVI version 8.1.

\section{CONTROL OBJECTIVES AND ALGORITHM DESCRIPTION}

\section{A. Control problem formulation}

When trying to maximize the ECH absorption as described in Sec. I, the signal we are trying to maximize is a function of the polarization but also of unknown and/or varying quantities in the plasma. From a control design perspective, it would be desirable to have measurements of as many quantities as possible which influence the ECH wave absorption. Then a controller based on an approximate model of the absorption process can use these parameters to take appropriate control actions. In practice, however, precise measurements of, for example, the edge density and location (among others) are not readily available in real time; thereby precluding the calculations required to set the optimum polarization. These considerations have led to the choice of a simpler control strategy, which is to implement a search routine rather than to perform a model-based controller design.

Furthermore, it must be noted that the mapping between the polarizer mirror position and the resulting polarization is a (nonlinear) static map which does not involve any dynamics. The part of the system which can be considered dynamic - the response of the polarizer mirrors to motor commands - is taken care of by the motion controller and does not need to be considered in the control algorithm design. Also, due to the maximum rotation speed of $2 \%$ and typical angle changes of $\sim 1^{\circ}$, the time involved in moving the mirrors is much larger than that involved in calculations and the computation time and transmission delays can be neglected. For these reasons it was not necessary to take any system dynamics into account, and a measurement of the time evolution of the quantity we are attempting to optimize was deemed sufficient. In other words, the polarizer position is not included in the feedback path.

We have described the control problem in the case where the objective is maximization of the ECH absorption. However, in a different type of plama experiments it may be 
desirable to set and track a given polarization of the $\mathrm{ECH}$ beam, expressed by the polarization angles $\alpha$ and $\beta$ (respectively, indicating the linear and elliptical polarization angles, see Sec. IV for details). A system for measuring these polarization angles in real time is installed in the LHD ECH system transmission line. ${ }^{7}$ Below it will be shown how a similar search algorithm as used for absorption optimization can be used for this application as well.

Both applications described above can be cast in the form of a minimization search routine of a particular cost function. The choice of this cost function depends on the particular application envisioned. When the objective is to maximize the ECH absorption efficiency, the cost function can be taken as the negative of a signal indicating this absorption. One could think of, for example, the core temperature derived from electron cyclotron emissions (ECE temperature) or the variation in measured ECE temperature while modulating the gyrotron power,

$$
J=-\left\langle\Delta T_{\mathrm{ECE}}\right\rangle .
$$

To simulate changing plasma conditions in the low-power setup described in Sec. II, we chose to take the voltage on one of the detectors as the signal to be maximized. The appropriate cost function to be minimized then simply is

$$
J=-\left|V_{\text {detector }}\right| .
$$

When tracking a reference polarization, it is natural to select a cost function corresponding to some error norm. For example, one may choose

$$
J=\left(\alpha-\alpha_{\mathrm{ref}}\right)^{2}+\left(\beta-\beta_{\mathrm{ref}}\right)^{2} .
$$

However, it should be noted that for purely circular polarization $\left(\beta=45^{\circ}\right)$ the linear polarization angle $\alpha$ is arbitrary and plays no role. Therefore a better choice would be

$$
J=f(\gamma)\left(\alpha-\alpha_{\mathrm{ref}}\right)^{2}+\left(\beta-\beta_{\mathrm{ref}}\right)^{2},
$$

where $\gamma=\left(1-|\beta| / 45^{\circ}\right)$. A suitable choice of $f(\gamma)$, with $f(0)$ $=0$, lets the importance of $\alpha$ decay as the polarization comes closer to being circular.

Naturally, when the objective is to control only the linear polarization $(\beta=0)$, one can choose simply

$$
J=\left(\alpha-\alpha_{\text {ref }}\right)^{2} .
$$

Typically, for nonperpendicular incidence the polarizers are nonideal and each affect both $\alpha$ and $\beta$ simultaneously. It is therefore beneficial to include both measurements in the algorithm if available. In Sec. IV a number of the above cost functions is used in different experiments.

\section{B. Cyclic coordinate search algorithm}

In this subsection, the minimization search algorithm that is utilized will be described. In its simplest form, the algorithm moves along one dimension only (corresponding one polarizer plate angle) until it encounters a local minimum in the cost function.
1. Start at position $x$ with cost function $J(x)$ and initial

search coefficient $r=1$

2. Move from initial position $x$ over a distance $r d$

3. Evaluate $J(x+r d)$

4. If $J(x+r d)<J(x)$ then $x=x+r d$, goto 2

5. If $J(x+r d)>J(x)$ then

- if this is the first iteration then reverse direction.

$r=-1$, goto 2

- else go back to local minimum $x$, exit.

The parameter $d$, the minimum distance the algorithm moves before re-evaluating the cost function, has to be tuned appropriately. Setting it too large will make the algorithm unnecessarily inaccurate. Setting it too small makes the algorithm more sensitive to noise which can create "fake" local minima.

The one-dimensional search algorithm described above can be extended to more dimensions by repeating the algorithm for each movement coordinate cyclically. If the cost function is well behaved, in the sense that it has no unwanted local minima, the algorithm will converge to the "true" local minimum after some iterations. The algorithm will continue to search around the found minimum regardless of the quality of this minimum. This is to ensure that the minimum can be tracked if its location changes in time. Another straightforward extension of this algorithm is to calculate and store the minimum value of $J(x)$ at intermediate values of $x$ during the motion from one point to another. If the algorithm has completed it will go back to an intermediate $x$ corresponding to the minimum value of $J(x)$ that it had found and the search will restart from there.

This algorithm is, in fact, a simple form of a so-called hill-climber algorithm ${ }^{8}$ which is routinely used for simple problems in nonlinear numerical optimization. It is particularly useful for this problem since it is easiest and fastest to evaluate only points in the cost function landscape which lie close to the current point (since the polarizers have to move for every new evaluation). This rules out more complex nonlinear optimization methods such as the golden section method. ${ }^{8}$ It should be noted that most of the more sophisticated search routines require at least some knowledge of the function being optimized, such as the value of the local gradient or Hessian. Although it would in principle be possible to estimate these from the measurements, it was decided to use the hill climber for simplicity.

Section IV describes the results obtained by testing the algorithm described above for signal maximization and linear polarization angle tracking.

\section{RESULTS}

\section{A. Maximization of crystal detector voltage}

The first and simplest feedback control algorithm which was tested in the NIFS low-power test stand attempts to maximize the voltage from one of the two orthogonal crystal detectors. As described in Sec. II, the receiver assembly can be rotated using the third rotating stage. This was used to see the response of the control algorithm to changes in the location of the cost function optimum. As has already been men- 

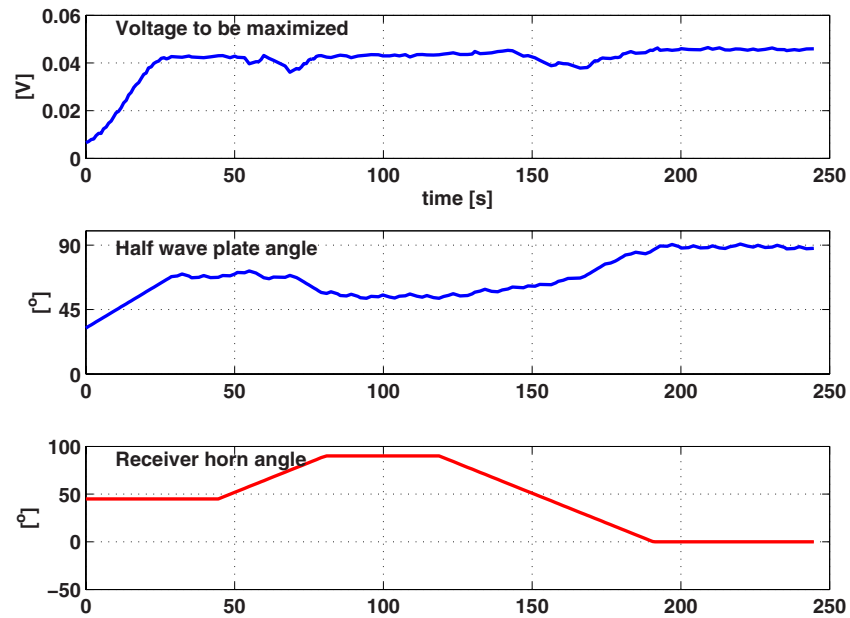

FIG. 2. (Color online) Demonstration of one degree of freedom receiver voltage maximization. Only the half-wave plate controlling the linear polarization angle is varied in this experiment. Top: The voltage of the receiver, corresponding to the signal the control algorithm tries to maximize. Center: The actual movement of the half-wave plate commanded by the controller. Bottom: Rotation of the detector assembly. After each rotation a different polarization is required for maximum voltage, so the controller has to move the polarizing mirrors to compensate.

tioned, this can be thought of as a similar situation as plasma absorption maximization. Equation (2) was chosen as the cost function.

Tests were done at first with only the half-wave plate $(\lambda / 4)$ rotating while the quarter-wave plate $(\lambda / 8)$ angle is set to zero. In ideal conditions this corresponds to zero ellipticity, but because of the $45^{\circ}$ incidence angle in this setup there will be some elliptical polarization as well. The step size for the search algorithm was set to $d=1^{\circ}$. While the algorithm was running, the receiver horn was rotated to different positions from time to time. The result of this experiment is displayed in Fig. 2. As can be observed, the algorithm initially moves the half-wave plate to increase the receiver voltage. Once a maximum is reached, the half-wave plate angle remains constant. Subsequently, the controller responds to the change in the receiver horn position and attempts to increase the measured receiver voltage. The maximum voltage is similar in all situations $(t=10,100,200 \mathrm{~s})$, suggesting that there are no problems related to local minima. After moving to a certain position, The half-wave plate angle is seen to be oscillating around this position (e.g., $t>200 \mathrm{~s}$ ). This is because the algorithm continues to move in an attempt to detect a change in the location of the maximum. It must be noted that in some cases (not displayed) the control system did not respond promptly to the varying receiver horn position. This behavior is attributed to the fact that the search algorithm is designed to search in a fixed cost function landscape. When the landscape changes on a time scale similar to the search speed the performance may not be the same as when the landscape is fixed. This can be improved by modifying the search algorithm to be able to account for such situations.

In a second experiment, the quarter-wave plate was allowed to move as well. The control algorithm then sequentially searches for a local minimum in each direction (cyclic coordinate search). Again, the step size was fixed to $1^{\circ}$. The results are shown in Fig. 3. As can be observed, the extra
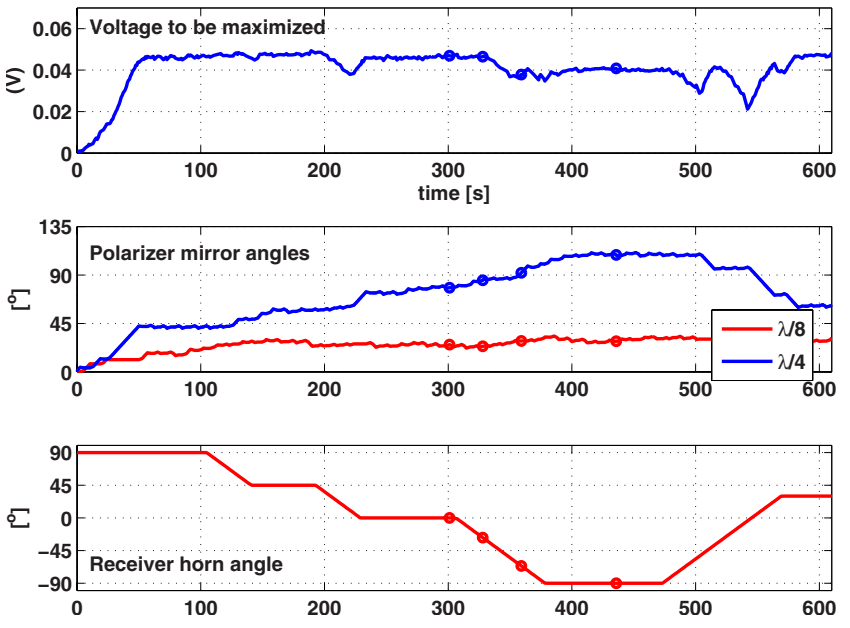

FIG. 3. (Color online) Demonstration of two degree of freedom receiver voltage maximization. Both the half-wave plate and the quarter-wave plate are allowed to rotate. The additional degree of freedom offered by the quarter-wave plate allows to obtain a higher voltage than the one degree of freedom case shown in Fig. 2. The circles indicate the times which are analyzed further in Fig. 6.

freedom is used since the quarter-wave plate moves away from its zero position. Yet, most of the optimization involves the half-wave plate $(\lambda / 4)$ only. This is to be expected since it is the half-wave plate which controls the linear polarization angle, and the latter should be aligned with the detector in order to maximize the receiver voltage. Still, it should be noticed that the maximum voltages reached during this demonstration are not always the same.

To explain the results of this experiment, calculations were done based on the physics of polarizer grating mirrors. Equations for the complex-valued Jones vector, ${ }^{9}$ which describes the polarization of the reflected wave as a function of the incoming wave and the polarizer mirror properties for the geometry in Fig. 4 can be written as ${ }^{10}$

$\left(\begin{array}{c}E_{\phi, r} \\ E_{\theta, r}\end{array}\right)=\left[\begin{array}{cc}\cos (\xi) & -\sin (\xi) \\ \sin (\xi) & \cos (\xi)\end{array}\right] \cdot\left(\begin{array}{cc}-e^{i \tau / 2} & 0 \\ 0 & e^{-i \tau / 2}\end{array}\right) \cdot\left[\begin{array}{cc}\cos (\xi) & -\sin (\xi) \\ \sin (\xi) & \cos (\xi)\end{array}\right] \cdot\left(\begin{array}{c}E_{\phi, i} \\ E_{\theta, i}\end{array}\right)$,

$$
\xi=\tan ^{-1}[\cos (\theta) \tan (\phi)] .
$$

Here $\theta$ is the angle between the polarizer mirror surface normal and the incoming wave propagation vector $\boldsymbol{\kappa}$ and $\phi$ is the rotation angle of the polarizer mirror. $\tau$ represents the phase difference between the reflected components parallel and perpendicular to the mirror gratings. For ideal mirrors of grating depth $g$ this is given by $\tau=4 \pi g / \lambda$. For nonideal grat-

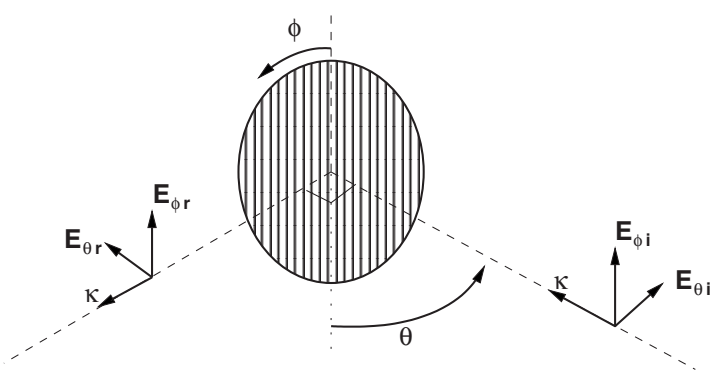

FIG. 4. Coordinate system definition for Eqs. (6)-(8). $\phi=0$ corresponds to vertical gratings. 


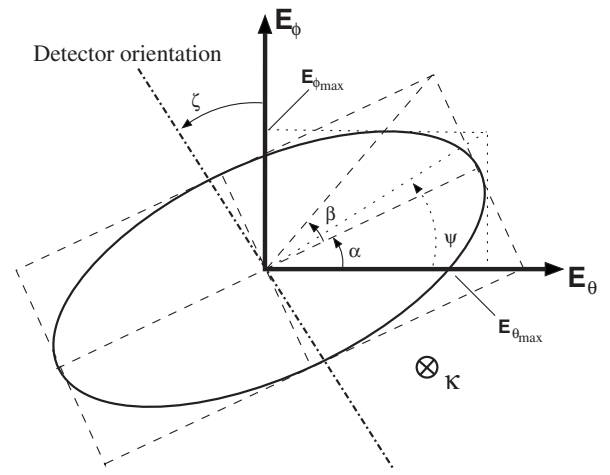

FIG. 5. Illustration of angles used to describe polarization. Polarization angles $\alpha$ and $\beta$ define the major and minor axes of the ellipse described by the electric field vector. The ratio between the electric field components $\psi$ is approximately equal to $\alpha$ for small values of $\beta$.

ings an expansion in even cosine terms of the form

$$
\tau=\sum_{n=0}^{N} m_{i} \cos (2 n \phi)
$$

is often employed, where the coefficients can be determined for each grating independently. The polarization angles are defined in terms of the electric field components as ${ }^{9}$

$$
\tan \psi=\frac{E_{\phi, \max }}{E_{\theta, \max }},
$$

$\tan 2 \alpha=(\tan 2 \psi) \cos \delta$,

$\sin 2 \beta=(\sin 2 \alpha) \sin \delta$,

where $\delta$ is the phase difference between the two orthogonal electric field components.

Using these equations we can calculate the expected voltage of one of the crystal detectors if we take into account the degree of power coupling between the input beam and one channel of the orthomode transducer at a given angle,

$V_{r} \sim \cos \left(\alpha-\alpha_{\text {det }}\right)^{2} \cos (\beta)^{2}+\sin \left(\alpha-\alpha_{\text {det }}\right)^{2} \sin (\beta)^{2}$,

where $\alpha, \beta$ are the polarization angles of the beam entering the receiver and $\alpha_{\mathrm{det}}=\pi / 2+\zeta$, with $\zeta$ the orientation angle of the receiver assembly rotating stage. The equation above is derived from the projection of the electric field component on the detector orientation axis. See Fig. 5 for details.
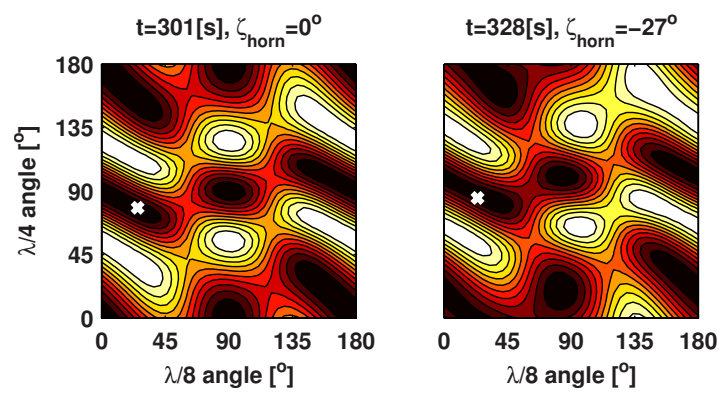

From the expression for $\tau$ for nonideal polarizers one can see that a nonideal half-wave plate can also add some amount of elliptic polarization to the reflected wave. Therefore, $\alpha$ and $\beta$ can never be varied completely independently of each other. Since ellipticity (nonzero $\beta$ ) will result in lower than optimal coupling [Eq. (12)] it can be understood that complete coupling may not be obtained in all cases. This helps to explain the different levels of the maxima reached during the experiment. Additionally, nonideal behavior of the orthomode transducer can also affect the results.

In Fig. 6, the behavior of the controller between $t$ $=300 \mathrm{~s}$ and $t=420 \mathrm{~s}$ is illustrated. The voltage landscape, calculated from the theoretical model described above, is plotted as a function of the two polarizer angles. Dark areas indicate regions where the voltage is high (thus the cost function is low). This landscape changes in time due to the rotation of the detector assembly. The measured position of the polarizers is displayed as a white cross: the controller adjusts the position of the polarizers in order to follow the movement of the region of maximum voltage.

As can be seen, the theoretical voltage landscape has several local maxima and due to the nonideal characteristics of the gratings the local maxima will not all be identical. Indeed, the local maximum which the algorithm finds is not necessarily the global maximum. Additionally, because only one polarizer is moved at a time only vertical or horizontal movements on the cost function landscape are possible. Therefore if the path to a local maximum is diagonal it will be difficult to follow. These kinds of limitations are difficult to avoid in a local search algorithm such as the one used here, as it is assumed that we do not have an a priori knowledge of the landscape. More complex search routines are a topic of future study.

\section{B. Linear polarization control}

In a different type of experiment, attempts were made to control the linear polarization directly by specifying a reference value. In this setup, the detector assembly was not rotated but placed at $\zeta=0$ such that one detector measured the vertical component of the incoming wave and the other measured the horizontal component. In this experiment the quarter-wave plate was kept fixed at $0^{\circ}$, so the wave was
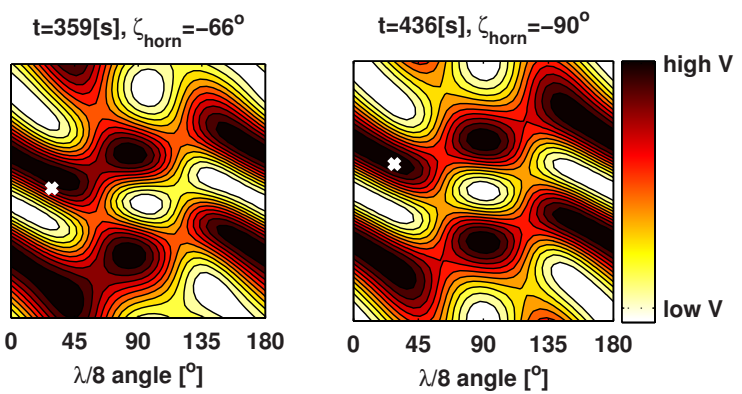

FIG. 6. (Color online) The behavior of the controller between $t=300 \mathrm{~s}$ and $t=420 \mathrm{~s}$ is illustrated in this figure. The cost function landscape, calculated from a theoretical model, is plotted as a function of the two polarizer angles. Dark, red areas indicate regions where the voltage is high (thus the cost function is low). This landscape changes in time due to the rotation of the detector assembly. The measured position of the polarizers is displayed as a cross. As can be observed, the controller adjusts the position of the polarizers in order to follow the movement of the region of maximum voltage. However, it is clear that several local maxima exist. The algorithm will try to move to a nearby local maximum, which is not necessarily the global optimum. 

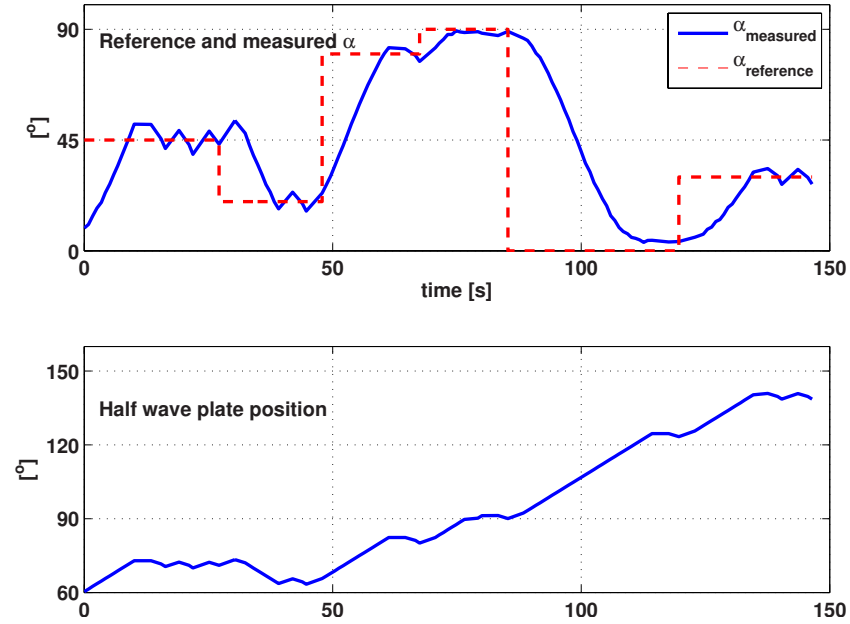

FIG. 7. (Color online) Demonstration of linear polarization angle tracking. Top: The measured linear polarization angle $|\alpha|$ (blue-solid) successfully tracks the reference value (red-dotted). The oscillations are due to the nature of the control algorithm which continues to move in search of changes in the cost function minimum location. Bottom: The half-wave plate angle, controlled by the control algorithm. Note that the $\lambda / 4$ plate position increases while $|\alpha|$ can both decrease or increase. This is because of the fact that phase information is lost in the measurement, and it is not possible to distinguish positive from negative values of $\alpha$.

(ideally) linearly polarized only. With this setup the apparent linear polarization angle can simply be calculated from the receiver voltages as

$$
\alpha \approx \psi=\operatorname{atan}\left(\frac{\left\langle E_{\phi, \text { out }}^{2}\right\rangle}{\left\langle E_{\theta, \text { out }}^{2}\right\rangle}\right)=\operatorname{atan}\left(\frac{V_{1}}{V_{2}}\right),
$$

where all the phase delay information has been lost. Note that the above expression is only valid for small values of $\beta$ and $0<\alpha<\pi / 2$. For details, see Ref. 9.

The reference polarization angle $\alpha_{\text {ref }}$ is varied several times during the experiment, and as can be observed in Fig. 7, the control algorithm adjusts the half-wave plate in order to obtain this angle. When the angle is reached the search continues, so an oscillation around the target value can be observed. As mentioned before, there is a trade off between the degree of oscillation around the reference value and the sensitivity of the algorithm to measurement noise. It should be noted that when the half-wave plate angle exceeds $90^{\circ}, \alpha$ jumps from $90^{\circ}$ to $-90^{\circ}(\alpha$ is only defined from $-90<\alpha$ $\leq 90$ ). However, because the phase information has been lost in the measurement, $\alpha$ will only be measured between $0^{\circ}$ and $90^{\circ}$. Therefore it is actually $|\alpha|$ which is being controlled in this experiment. This can be avoided either by including phase information in the measurement or by restricting the operating regime of the polarizer plates to cover only the region where there is a one-to-one mapping between polarizer plate angles and measured $\alpha$.

It should also be mentioned that this experiment could in principle readily be extended to two degrees of freedom to allow the control of $\beta$ as well as $\alpha$ provided a measure of $\beta$ is available. This also requires information about the relative phase of the electric fields, which was not available in the setup used.

In all the experiments described above, the characteristic time to reach the cost function minimum was of the order of 10-20 s. By examining the curves of the half-wave plate movements it becomes clear that this is due to the saturation of the polarizer rotation speed at $2 \%$, mentioned in Sec. II. Overall, a new target position for the polarizers is computed approximately once every second. This is determined by the time it takes the rotating stages to move the requested distance. This also confirms that compared to these time scales the computation time is negligible; therefore, there would have been little to gain from using specialized real-time hardware and software.

\section{CONCLUSIONS AND FUTURE WORK}

The proof of principle presented in this article shows that it is possible to control the polarization of an ECH beam in real time using feedback control. It has been shown that a single cost-function based approach was effective in controlling a nonlinear function of the polarizer angle settings. An application of this is the maximization of $\mathrm{ECH}$ absorption in a fusion plasma. A control algorithm has been designed which relies on a simple hill-climbing algorithm to minimize the cost function. This controller has been tested in a lowpower setup using two polarizing mirrors mounted on rotating stages, with crystal detectors measuring the orthogonal components of the reflected wave. Both maximization of the voltage on one of the detectors and tracking of a reference linear polarization angle are demonstrated. It is also shown that it is possible to follow a changing reference and cost function landscape.

New tests with faster polarizers and more sophisticated search methods are planned future work at NIFS as well as eventual implementation of real-time feedback control of polarization on the LHD ECH system.

${ }^{1}$ T. Stix, Waves in Plasmas (Springer, New York, 1992).

${ }^{2}$ T. Morisaki, K. Narihara, S. Masuzaki, S. Morita, M. Goto, A. Komori, N. Ohyabu, O. Motojima, K. Matsuoka, and L. E. Group, J. Nucl. Mater. 313, 548 (2003).

${ }^{3}$ I. Fidone and G. Granata, Nucl. Fusion 11, 133 (1971).

${ }^{4}$ T. Bigelow, Ph.D. thesis, University of Tennessee, 1990.

${ }^{5}$ S. Kubo, T. Shimozuma, Y. Yoshimura, H. Igami, Y. Mizuno, S. Ito, S. Kobayashi, Y. Takita, and T. Mutoh, Fusion Eng. Des. 83, 256 (2008).

${ }^{6}$ J. Doane and R. Olstad, Fusion Sci. Technol. 53, 39 (2008).

${ }^{7}$ T. Notake, H. Idei, S. Kubo, T. Shimozuma, Y. Yoshimura, S. Kobayashi, Y. Mizuno, S. Ito, Y. Takita, K. Ohkubo, W. Kasparek, T. Watari, and R. Kumazawa, Rev. Sci. Instrum. 76, 023504 (2005).

${ }^{8}$ W. H. Press, W. T. Vetterling, S. A. Teukolsky, and B. P. Flannery, Numerical Recipes, 2nd ed. (Cambridge University Press, New York, 1996).

${ }^{9}$ M. Born and E. Wolf, Principles of Optics (Pergamon, New York, 1974).

${ }^{10}$ F. Smits, Rijnhuizen Report No. 91-208, FOM, The Netherlands 1989. 